\title{
Enumération des Cestodes du plancton et des Invertébrés marins
}

\section{( $7^{\bullet}$ Contribution)}

par Robert Ph. DOLLFUS

\section{A-G Formes libres dans le plancton. \\ H-M Formes observées dans divers Invertébrés.}

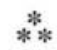

A) Dans le plancton, en liberté.

Nombreux sont les ouvrages qui traitent du zooplancton marin : bien peu relatent que l'on peut y trouver des œufs de Cestodes, des coracidies donnant des oncosphères, des larves de divers âges et même des adultes, sortis de leur hôte après la mort de celui-ci, ou expulsés avec les fèces. Dans ma publication de 1923, p. 276-278, j'ai résumé le peu de connaissances acquises sur ce sujet depuis l'observation par E. Claparède (1863) de deux individus d'une larve du type «Scolex» (Plerocercoïdes sp.) dans le plancton de surface, l'un du Firth of Clyde, l'autre de Saint-Vaast-la-Houghe (Manche).

Je peux, aujourd'hui, apporter une contribution importante à la connaissance de formes libres de Cestodes qui, avant d'atteindre l'état adulte, vivent dans le plancton.

Le triage à la loupe binoculaire (1) d'un prélèvement de plancton de surface (2) (pêche à la lumière par Théodore Monod) à Boavista (Iles du Cap-Vert), 27 octobre

(1) Par mon assistante technique, Mlle Janine Laurent, que je remercie pour le soin qu'elle a apporté à ce triage.

(2) Il s'agit d'un plancton à Euphausiacea, où l'espèce dominante est Nyctiphanes couchi (Bell 1853) (Henri Nouvel determin.). En moindre abondance dans ce plancton, il y a des Copépodes, des Isopodes, des Amphipodes, des Cumacés, des larves de Crustacés Décapodes, des Crustacés de divers autres groupes, des larves des Gastropodes, quelques larves de Téléostéens, des métacercaires libres de Distomes, des Nématodes libres, etc... 
1948, a montré la présence d'un grand nombre de larves et postlarves (3) de Tétrarhynques, de quelques postlarves de Tétraphylles et de chaînes de proglottis immatures ayant perdu leur scolex (fig. 34).

Il est vraisemblable que ces larves et postlarves sont libérées dans le plancton par la mort de petits Crustacés planctoniques, mais ce n'est pas absolument certain. Elles continuent à évoluer dans le plancton, s'accroissent, forment des proglottis. Que deviennent-elles? Jusqu'à quel stade continueront-elles cette croissance en liberté ? Parviennent-elles passivement dans un hôte planctonique ? Cela n'est pas encore connu.

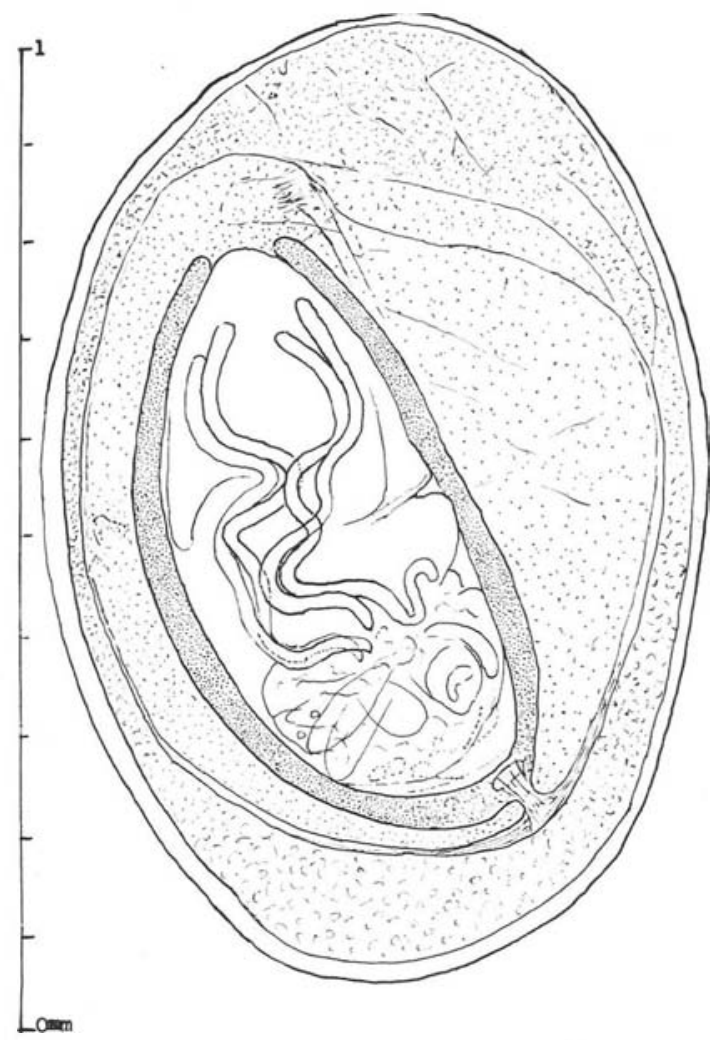

Fig. 1. - Larve de Tétrarhynques dans un blastocyste ovale. Plancton à la lumière, Boavista (Iles du Cap Vert).

(3) Je rappelle que je considère comme «postlarves» de Tétrarhynques et de Tétraphylles, les formes jeunes ayant abandonné ou perdu toute formation larvaire. N'ayant plus de blastocyste, elles ont dépassé le stade plérocercus ou plérocercoïde, elles ont déjà le scolex de l'adulte, se continuant par un prolongement caudal (représentant l'ébauche du strobile) à partir duquel les proglottis seront proliférés. Il arrive que des postlarves libres, pendant leur vie planctonique, produisent déjà des proglottis, mais ceux-ci restent immatures. 
A 1) Plérocerques de Tétrarhynques encore pourvus de leur étui larvaire ou EN VOIE DE L'ABANDONNER.

Pour le plus grand nombre, les larves de Tétrarhynques du plancton que j’ai examinées sont encore pourvues de leur étui larvaire, quelques-unes sont en voie de l'abandonner et quelques autres l'ont récemment abandonné.

Les plus jeunes des larves appartenant certainement à des Tétrarhynques sont de forme ovale (mesurant, par exemple, $0,47 \times 0,31 \mathrm{~mm}$ ), n'ayant pas encore leur blastocyste formé ; il n'y a pas de délimitation entre la partie externe qui donnera les parois du blastocyste et la partie interne qui donnera le corps larvaire ; néanmoins, on distingue des ébauches de bulbes et quelques crochets commencent à apparaître.

Lorsque les larves sont devenues plus âgées et ont atteint une taille supérieure, le blastocyste est formé et sa cavité interne contient, replié, le corps de la larve en formation. La larve de la figure 1 a un contour régulièrement ovale et mesure $1 \times 0,67 \mathrm{~mm}$ après léger aplatissement. L'enveloppe externe paraît complètement close. Plus en dedans, il y a une seconde enveloppe, plus mince, devenant indistincte antérieurement. L'espace entre ces deux enveloppes est rempli par un parenchyme très lâche, peu distinct, sauf postérieurement. J'interprète ces deux enveloppes comme étant les parois externe et interne du blastocyste. La cavité réceptaculaire ne présente pas d'espace libre et contient un sac longitudinalement allongé en forme d'œuf, mesurant $0,64 \times$ $0,35 \mathrm{~mm}$. Ce sac a une paroi épaisse de $0,024 \mathrm{~mm}$, il présente une ouverture antérieure et son extrémité postérieure porte un petit faisceau de fibres qui la rattache à la paroi interne de la cavité réceptaculaire du blastocyste. Ce sac est entièrement rempli par la pars antica scolecis (qui comprend pars bothridialis, pars vaginalis, pars bulbosa et pars postbulbosa) repliée sur elle-même. Les bothridies ne sont pas discernables, les bulbes et les gaines se recouvrent et ne sont pas distincts, les proboscis seuls sont bien visibles, mais, étant invaginés, la forme et la disposition des crochets ne sont pas discernables ; néanmoins, on voit qu'il y a des crochets de plusieurs formes et de plusieurs grandeurs: l'armement est pécilacanthe. Le sac ouvert antérieurement et fixé postérieurement représente la pars postica scolecis; à maturité, la pars antica scolecis sort de ce sac, se déplie et s'étend longitudinalement ; à mesure que se produit cette extension, la cavité virtuelle du sac disparaît, le sac se retournant en doigt de gant, la paroi externe devient interne se soudant à elle-même, la paroi interne devient la paroi externe de la pars postica et celle-ci reste temporairement fixée par son extrémité postérieure à l'enveloppe blastocystique en dégénérescence.

Les larves des figures 2-4 sont claviformes plus ou moins allongées.

Chez la larve de la figure 2 , qui mesure $0,77 \times 0,3 \mathrm{~mm}$, la paroi externe du blastocyste est encore complètement close. La paroi interne est dégénérée antérieurement. La cavité réceptaculaire est occupée, sauf antérieurement, par la pars antica scolecis repliée sur elle-même et mesurant $0,37 \times 0,255 \mathrm{~mm}$. Les diverses parties du scolex se recouvrent et on ne voit qu'un enchevêtrement de proboscis et de gaines, masquant les bulbes. Les proboscis étant encore invaginés, la forme et la disposition des crochets ne sont pas observables ; cependant, on remarque, à la base du proboscis, 
des crochets beaucoup plus petits que les autres et il semble que, dans tout le reste des trompes, il y a au moins deux formes de crochets. Les bothridies étant repliées, il y a incertitude sur leur nombre. Par l'extrémité de sa partie postérieure, le scolex est fixé au fond de la cavité réceptaculaire ou moyen d'un petit faisceau de fibres.

Chez les larves des figures 3-4, l'ébauche des bothridies est reconnaissable et il semble y en avoir deux. L'armement des proboscis invaginés dans les gaines n'est
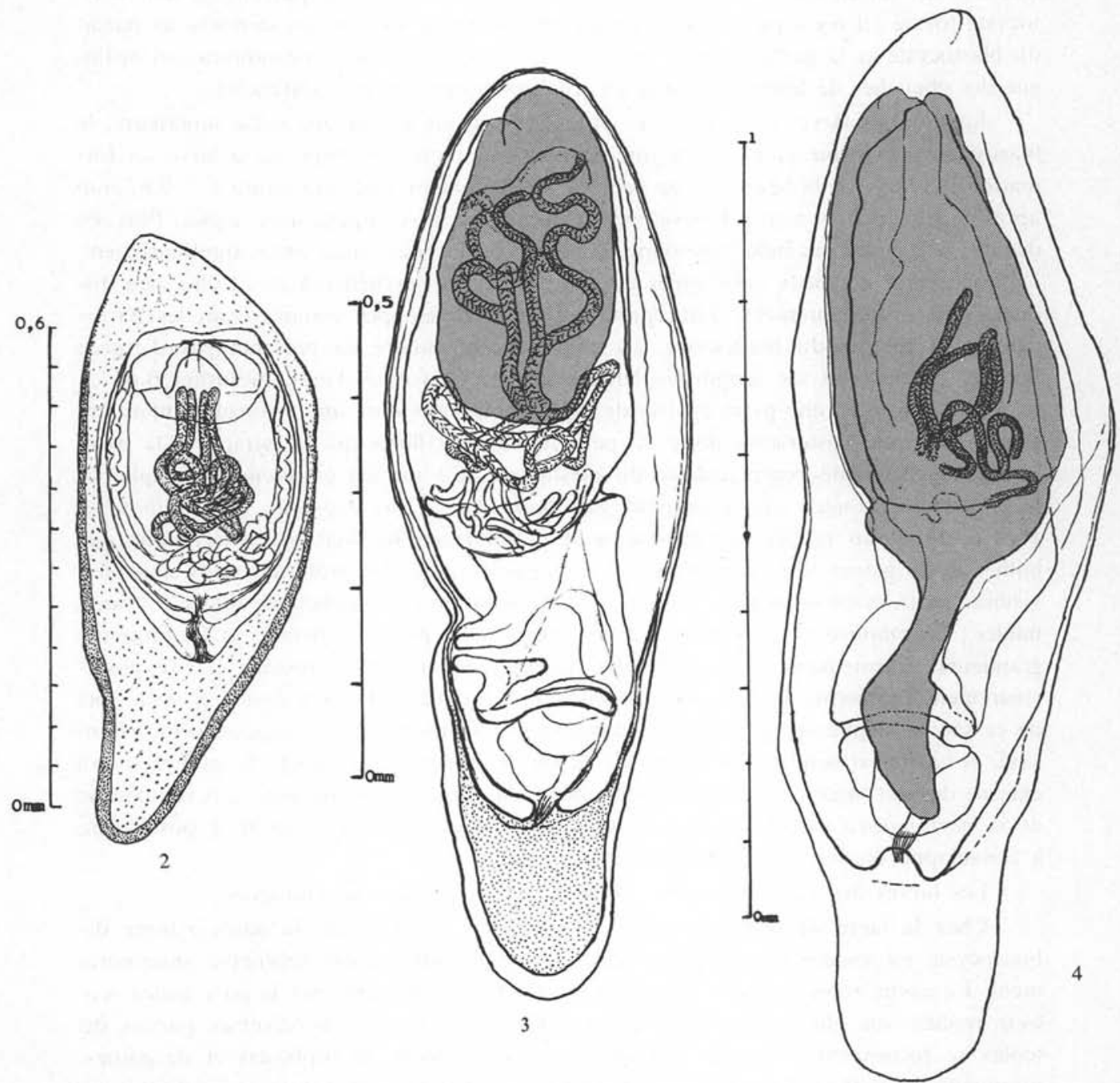

Fig. 2-4. - Larves de Tétrarhynques dans des blastocystes claviformes. Même provenance. 

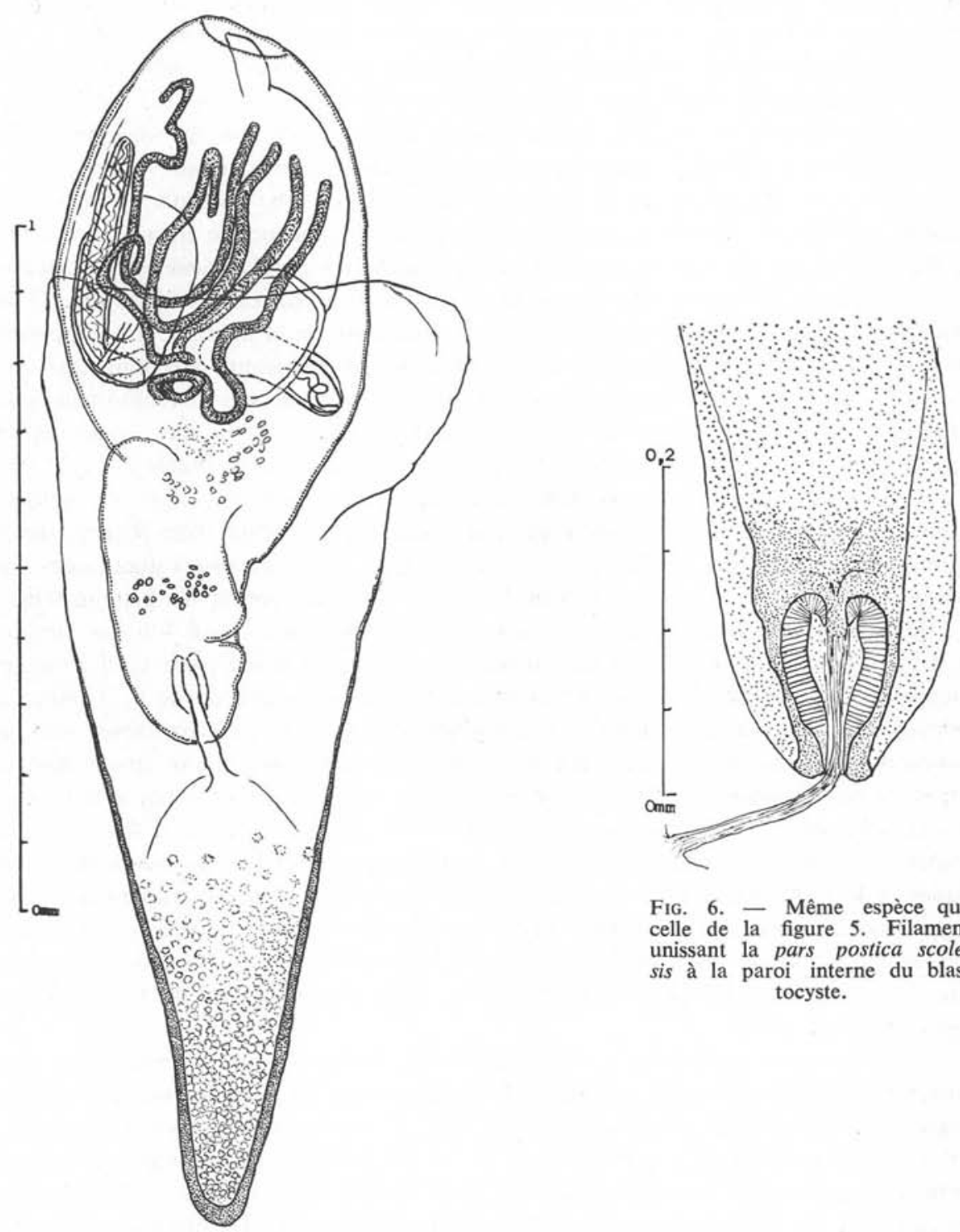

FIG. 6. - Même espèce que celle de la figure 5. Filament unissant la pars postica scolesis à la paroi interne du blastocyste.

Fig. 5. - Larve de Tétrarhynque se libérant de son étui larvaire. Même provenance. 
pas nettement observables. Les bulbes sont encore indistincts. L'attache du corps larvaire avec le fond de la cavité réceptaculaire du blastocyste est bien visible.

La figure 5 concerne un spécimen de larve dont j'ai examiné plusieurs exemplaires, les uns complètement enclos dans leur étui larvaire claviforme, les autres partiellement ou entièrement sortis de celui-ci. Aucun n'a son scolex étendu; ils ont tous leur scolex encore replié sur lui-même ; l'un d'eux, cependant, a commencé à étendre sa pars postica scolecis (fig. 5); il s'est en partie libéré de son étui larvaire, mais n'a pas rompu le filament fibrillaire le rattachant à la paroi interne de son blastocyste en dégénérescence. Ce filament sort d'une sorte d'ampoule ou de manchon à paroi épaisse, striée circulairement, situé dans la partie postérieure de la pars postica scolecis. Je figure (fig. 6) cet appareil particulier d'après un autre exemplaire que celui de la figure 5, mais apparemment conspécifique. Chez ce spécimen, le filament est beaucoup plus long que chez celui de la figure 5. A l'état replié, le scolex mesure $1 \mathrm{~mm}$ de son extrémité antérieure à son attache blastocystique, avec une plus grande largeur de $0,45 \mathrm{~mm}$. En raison des superpositions, aucune des parties du scolex n'est clairement discernable ; il y a peutêtre quatre bothridies. On ne voit nettement que les proboscis très longs, enchevêtrés, et une partie de deux des bulbes avec leur rétracteur s'insérant dans leur extrémité postérieure. La forme et la disposition des crochets ne sont pas observables ; ceux que l'on peut mesurer sont longs d'environ $20 \mu$, en forme de cornet un peu incurvé.

Le plerocercus de la figure 7 est encore complètement inclus dans son étui larvaire, où il est replié sur lui-même, ce qui empêche de connaître la longueur qu'il aurait en extension. J'évalue cette longueur à environ $1,95 \mathrm{~mm}$, sa largeur est de $0,23 \mathrm{~mm}$. Les bothridies ne sont pas discernables, la disposition des crochets n'est pas observable, les proboscis étant invaginés; ceux que l'on voit sans superposition ont une forme de cornet un peu incurvé avec une longueur d'environ $17 \mu$, sauf tout à fait à la base où ils sont plus petits et plus incurvés. Le diamètre des gaines est de $31 \mu$. Supposés rectilignes, les bulbes seraient longs de $0,40 \mathrm{~mm}$, larges de 0,055 ; ils comportent cinq coques et le rétracteur s'insère à leur extrémité postérieure. On remarquera que, chez ce spécimen, l'extrémité postérieure de la pars postica scolecis a rompu son attache avec la paroi interne du blastocyste, mais le corps de la larve est encore prisonnier dans son enveloppe, vestige de la paroi de la cavité réceptaculaire du blastocyste en dégénérescence.

Le plerocercus de la figure 8 a aussi une enveloppe claviforme ne présentant antérieurement aucune ouverture apparente. Cette enveloppe est plus épaisse à l'extrémité postérieure. La longueur totale est de $0,95 \mathrm{~mm}$, la plus grande largeur $0,25 \mathrm{~mm}$. La partie élargie correspond à peu près aux $2^{\circ}+3^{e}$ septièmes de la longueur totale et contient la pars antica scolecis, mesurant $0,32 \times 0,19 \mathrm{~mm}$. Celle-ci est repliée sur elle-même et les superpositions empêchent de voir clairement la forme et le nombre des bothridies, les dimensions des bulbes et l'armement des proboscis. On voit seulement que, sur une longueur d'environ $22 \mu$ à partir de la base, il y a une zone de crochets beaucoup plus petits. Il s'agit peut-être d'un genre de pécilacanthes, il ne s'agit évidemment pas d'un genre d'homéacanthes car, chez ceux-ci, les proboscis ne sont jamais aussi longs. 

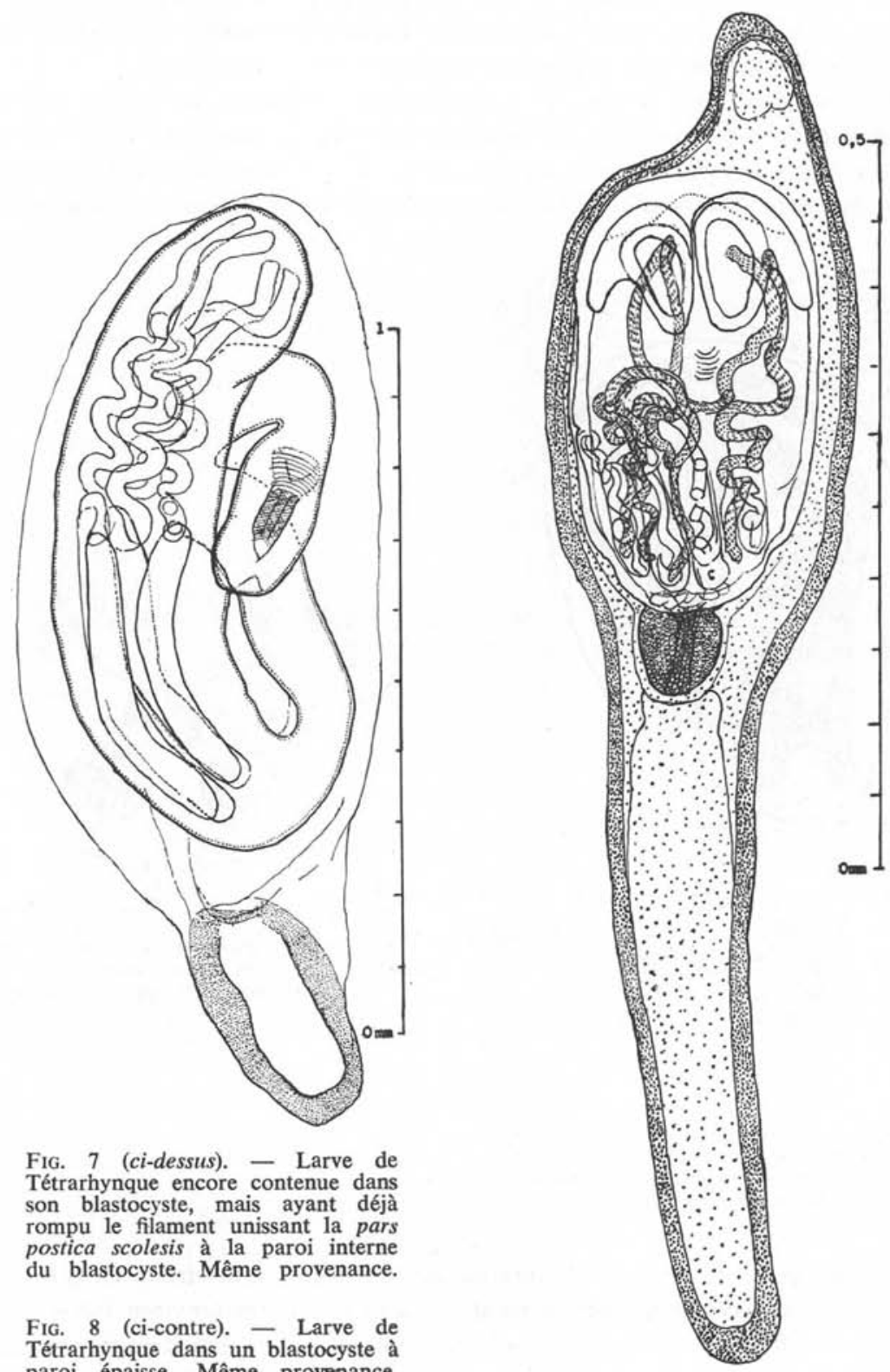

FIG. 8 (ci-contre). - Larve de Tétrarhynque dans un blastocyste à
paroi épaisse. Même provenance. 
Contre le bord postérieur de la pars antica scolecis, il y a une masse globuleuse de cellules plus colorables par le carmin dans laquelle s'irradient les fibres du filament réunissant le corps de la larve à la cavité réceptaculaire du blastocyste.

Du plerocercus des figures 9-11, j'ai examiné quelques individus, tous encore contenus dans leur étui larvaire. Le spécimen de la figure 9 mesure $0,90 \times 0,45 \mathrm{~mm}$. La pars antica scolecis est repliée dans la cavité réceptaculaire du blastocyste et ne se présente pas dans une condition favorable à l'observation. La pars bothridialis est en
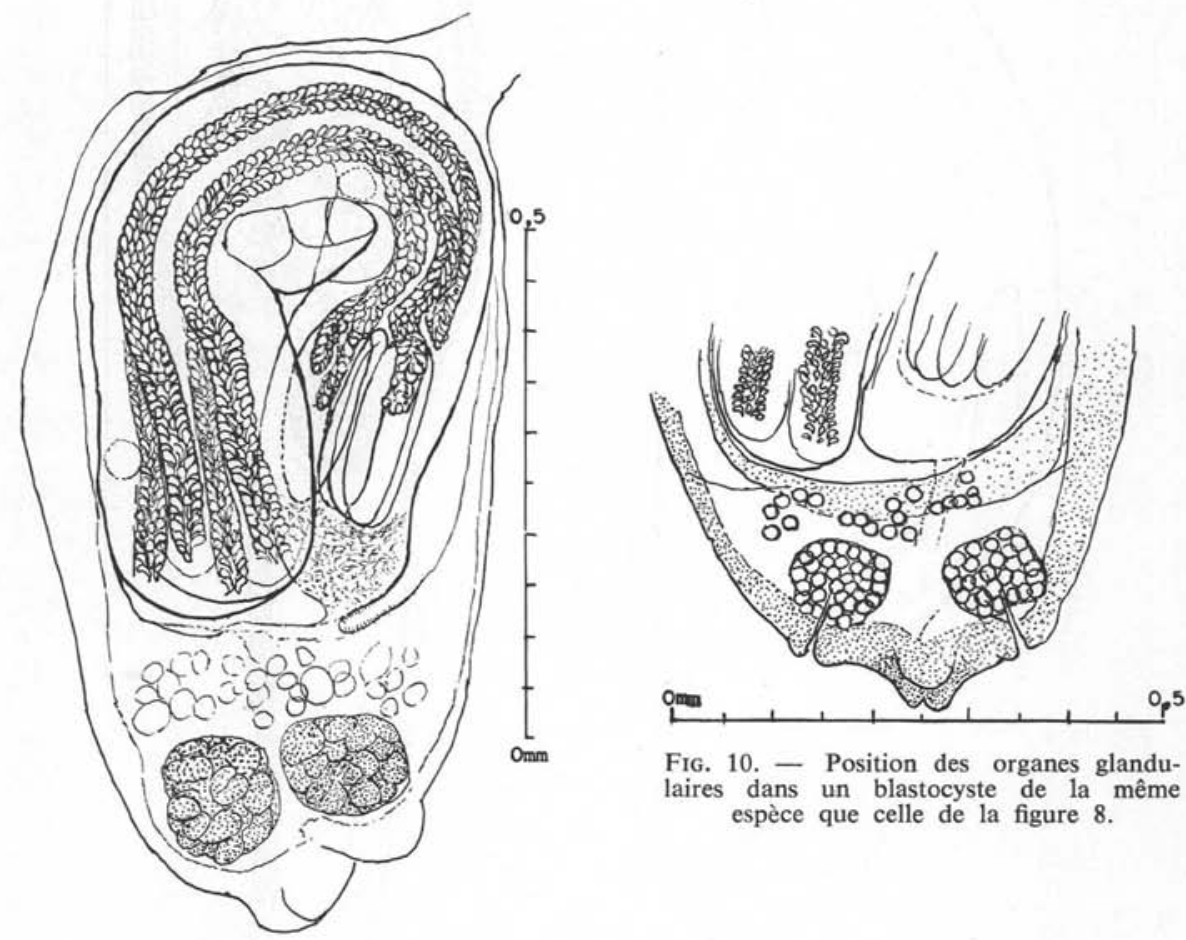

FIg. 10. - Position des organes glandulaires dans un blastocyste de la même espèce que celle de la figure 8 .

Fig. 9. - Plérocercus de Tétrarhynque dans un blastocyste pourvu postérieurement d'une paire d'organes glandulaires. Même provenance.

partie cachée et le nombre des bothridies est incertain ; peut-être y en a-t-il quatre. Les proboscis se recouvrent partiellement, ils sont très longs (environ $0,8-0,9 \mathrm{~mm}$ ) et portent de nombreuses rangées de crochets; comme ils sont entièrement invaginés, la dispositoin des crochets n'est pas reconnaissable. Il semble n'y avoir qu'une seule forme de crochets, tous en arceau, gros et courts (fig. 11) ; de profil, leur plus grande dimension est d'environ $17 \mu$; toutefois, ceux des extrémités sont un peu moins épais 


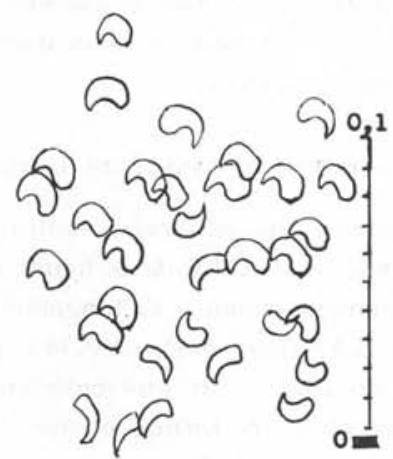

FIG. 11. - Quelques crochets d'un proboscis de la larve de la figure 8 .

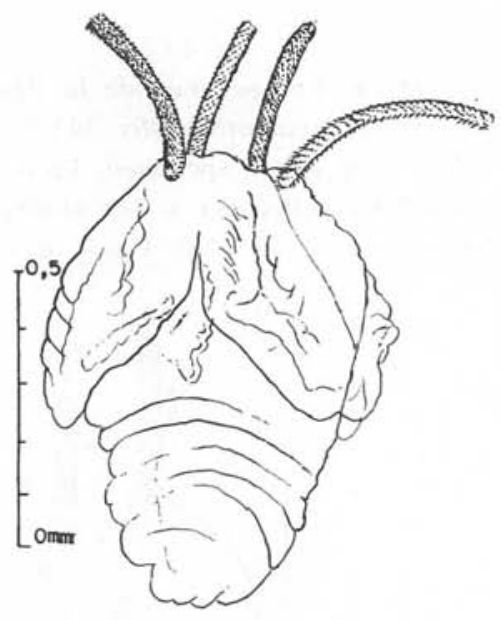

F1G. 13. - Postlarve de Nybelinia, $1^{\text {re }}$ forme. Même provenance.

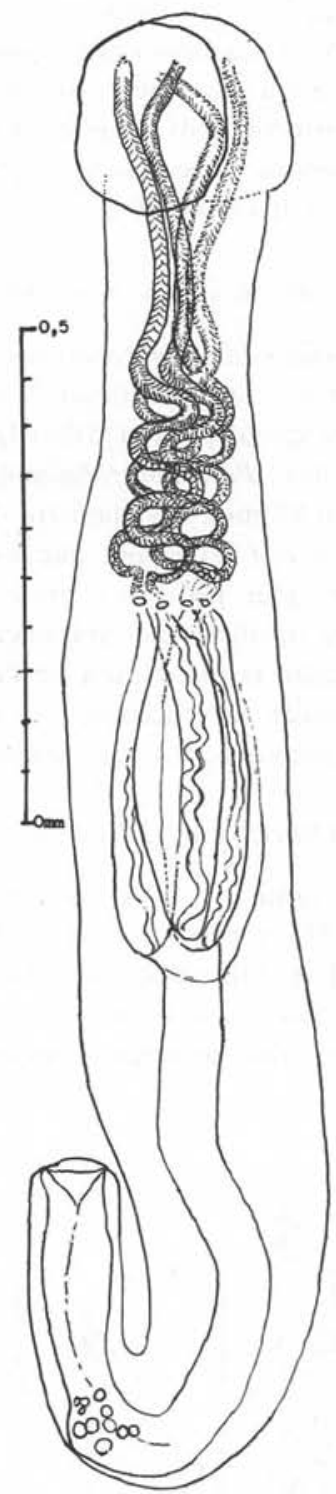

FIG. 12. - Larve de Tétrarhynque ayant abandonné son étui larvaire. Même provenance. 
et plus grêles. Les bulbes sont à peu près trois fois plus longs que larges (environ $196 \times 69 \mu$ ). La pars postbulbosa est occupée par la zone de prolifération, bien visible.

La partie postérieure du blastocyste contient deux gros amas de cellules glandulaires, formant deux glandes délimitées fortement colorables par le carmin. Chez un des spécimens, on constate que chacune de ces glandes est pourvue d'un canal évacuateur (fig. 10) s'ouvrant à l'extérieur de la paroi du blastocyste.

\section{A 2) Plérocerque de tétrarhynque observé seulement sans Étui larvaire.}

Plusieurs des plérocerques ci-dessus décrits ont été observés, tantôt contenus dans leur étui larvaire, tantôt l'ayant déjà abandonné, mais celui de la figure 12 n'a été rencontré que dépourvu d'étui larvaire et complètement étendu ; sa longueur totale est de $1,83 \mathrm{~mm}$. Pars bothridialis 0,2 , pars vaginalis 0,55 , pars bulbosa 0,348 , pars postbulbosa $0,88 \mathrm{~mm}$. Le diamètre des proboscis est de $16,6 \mu$; ils sont entièrement invaginés; on voit seulement que les crochets sont de plusieurs formes et que ceux de la base sont plus petits. Le bord postérieur libre des deux bothridies ne présente pas d'incisure médiane. Les rétracteurs des proboscis s'insèrent au fond des bulbes. Faute de connaître la disposition de l'armement des proboscis, on ne peut savoir qu'il s'agit d'une espèce hétéracanthe ou pécilacanthe; néanmoins, on remarque une certaine ressemblance avec les hétéracanthes à deux bothridies du genre Eutetrarhynchus.

\section{B) Postlarves de NYBELINIA.}

$1^{\text {re }}$ forme. - C'est une forme très courte, trapue. Le spécimen de la figure 13 mesure $811 \times 618 \mu$. Pars bothridialis $467,5 \mu$, pars metabothridialis 343,7 $\mu$. Les gaines et les bulbes ne sont clairement observables chez aucun spécimen. Le diamètre des proboscis, sans les crochets, est de $20 \mu$, avec les crochets $33 \mu$. Les crochets ont un faible talon antérieur, un assez long talon postérieur (fig. 14). Ceux de la face

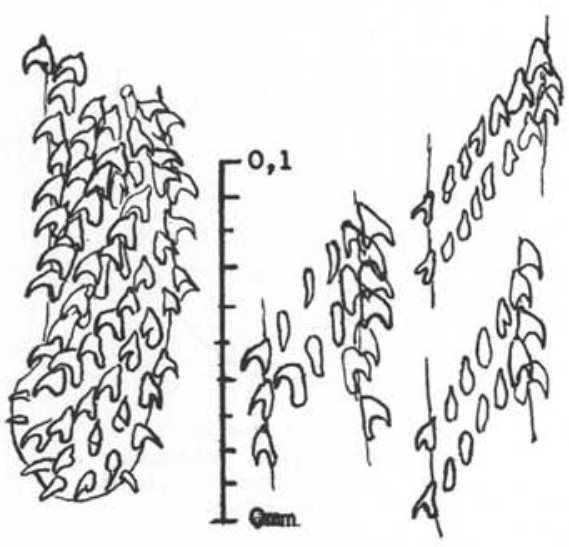

Fig. 14. - Armement des proboscis de la postlarve de la figure 13 .

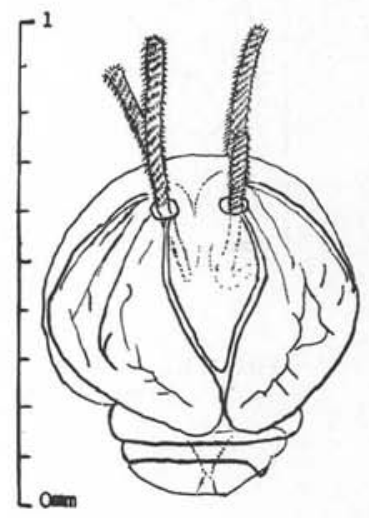

FIG. 15. - Postlarve de Nybelinia, $1^{\text {re }}$ forme. Même provenance. 
bothridiale mesurent 12 à $13,33 \mu$; ceux de la face antibothridiale 9,33 à $10,66 \mu$. Il n'y a pas d'armement basal particulier.

Le spécimen de la figure 15 mesure $0,68 \times 0,68 \mathrm{~mm}$. Le diamètre des proboscis est de 30 à $38 \mu$ (non compris les crochets); la hauteur des crochets de la face bothridiale est de $13,5 \mu$; sur la face antibothridiale, les crochets sont sensiblement plus petits, avec une hauteur de $8 \mu$ (du talon antérieur au talon postérieur) (fig. 16). Ils sont sur 16 files longitudinales.

Cette postlarve montre que la pars bothridialis atteint sa longueur définitive alors que la pars metabothridialis commence seulement à s'accroître. Cet accroissement a lieu au cours de la vie planctonique; j'ai, en effet, des spécimens à pars metabothridialis montrant cet accroissement progressif.

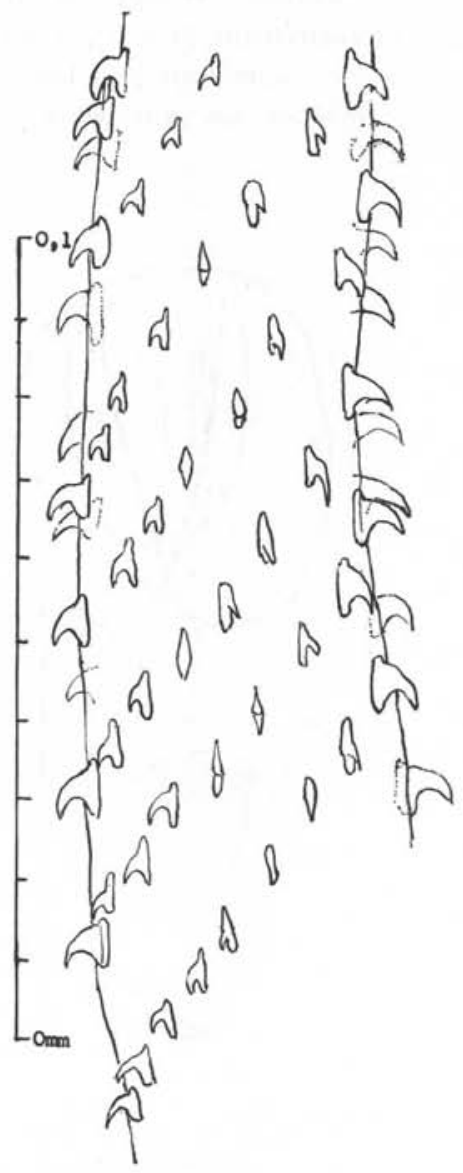

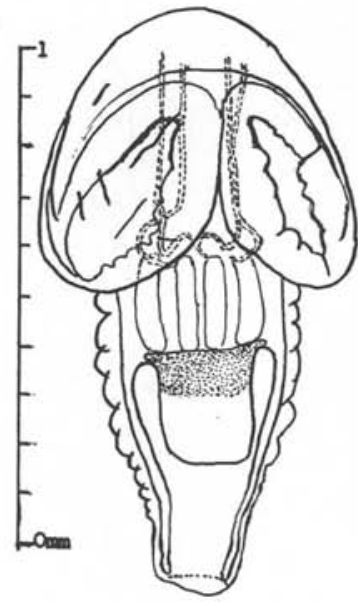

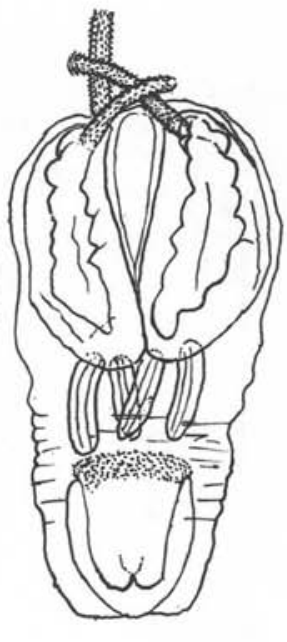

Fig. 17-18. - Postlarves de Nybelinia, 2e forme. Même provenance.

Fig. 16. - Armement d'un proboscis du spécimen de la figure 15 . 
Cette forme courte appartient au groupe d'espèces de Nybelinia de ma section V a (voir R.-Ph. Dollfus, 1960, p. 791), où j'ai décrit: dakari R.-Ph. D., estingmena R.-Ph. D., punctatissima R.-Ph. D. avec quelques variétés, mais elle ne correspond exactement à aucune de ces espèces.

$2^{\circ}$ forme. - Les spécimens de cette forme mesurent, en moyenne, $1 \times 0,6 \mathrm{~mm}$. La pars bothridialis est plus courte que la pars metabothridialis, ou égale (fig. 17-19). Les bothridies sont pourvues d'un large bourrelet marginal, dont le bord interne est irrégulièrement ondulé et il y a quelques sillons superficiels, çà et là, sur la pars bothridialis. Les bulbes mesurent, en moyenne, $264 \times 65 \mu$. L'appendix est long, en moyenne, de $0,2 \mathrm{~mm}$. Le velum est plus ou moins long selon les individus, il peut atteindre deux fois la longueur de l'appendix. Les proboscis ont un diamètre de $26,7 \mu$ sans les crochets, $43,7 \mu$ avec les crochets. Il n'y a pas de crochets particuliers à la base des proboscis, mais les crochets y sont un peu plus petits qu'antérieurement. Il y a dix files longitudinales de crochets, dont les plus grands ont 12 à $14 \mu$ dans leur plus longue dimension. Les plus grands crochets sont sur la face bothridiale, les plus petits, au

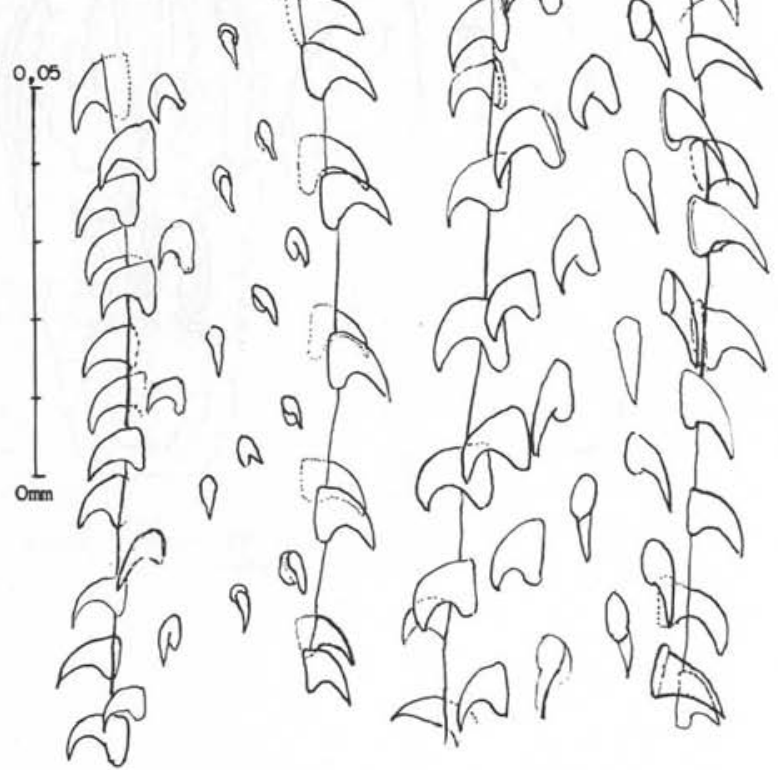

FIG. 19. - Armement d'un proboscis du spécimen de la figure 18 .

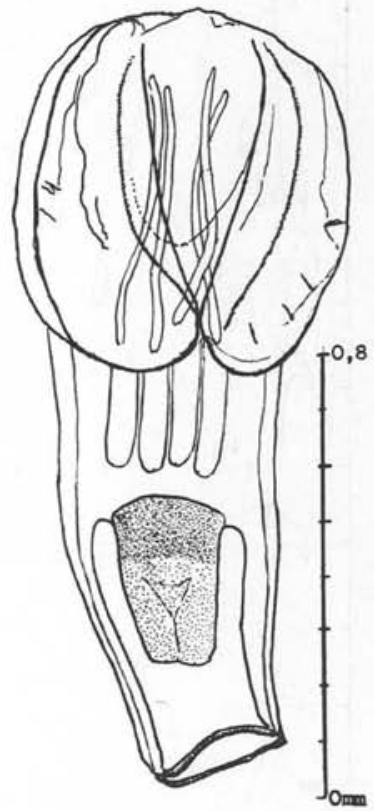

Fig. 20. - Postlarve de Nybelinia, $3^{\text {e }}$ forme. Même provenance. 
même niveau, sur la face antibothridiale. L'angle antérieur des crochets ne se prolonge pas par un talon.

Ce Nybelinia appartient au groupe d'espèces dont les proboscis n'ont pas d'armement basal particulier et dont les crochets antibothridiaux sont plus grêles que les bothridiaux, mais il ne correspond exactement à aucune des espèces de ce groupe.

$3^{\text {e }}$ forme. - C'est une forme moyennement allongée ; le spécimen de la figure 20 mesure $1,35 \times 0,59 \mathrm{~mm}$. Pars bothridialis 0,65 , pars metabothridialis 0,70 , pars bulbosa 0,07 , velum 0,46 , appendix $0,29 \mathrm{~mm}$. Le diamètre des proboscis invaginés est d'environ $14 \mu$, les crochets sont en arceau (fig. 21) et longs d'environ $8 \mu$, sauf à la base, où ils sont plus petits. Comme tous les proboscis sont complètement invaginés, il n'est pas possible de préciser leur nombre par tour de spire; il semble y en avoir environ douze.

\section{C) PSEUDONYBELINIA ODONTACANTHA R.-Ph. Dollfus 1966 (fig. 22-26).}

J'ai désigné sous ce nom (R.-Ph. Dollfus, 1966, p. 2612, 2615, fig. 1, 4) une postlarve de Tétrarhynque dont j'ai eu à ma disposition de nombreux individus prélevés dans le même plancton que celui contenant les larves et postlarves ci-dessus décrites.

Son habitus rappelle beaucoup celui des Nybelinia, mais c'est une forme à deux bothridies, alors que, par définition, les Nybelinia en ont quatre ; de plus, le bord pos. térieur libre de chaque bothridie est pourvu d'une fossette ciliée (fig. 24), dite « fossette sensorielle », comme chez les Otobothriens. La surface des bothridies montre des sillons sinueux irréguliers. Le spécimen choisi pour type a les dimensions ci-après : longueur totale $3,1 \mathrm{~mm}$, épaisseur maximum (au niveau de la pars bothridialis) $1 \mathrm{~mm}$; longueur de la pars bothridialis $1,47 \mathrm{~mm}$; diamètre des gaines et des proboscis (sans les crochets) $66 \mu$; diamètre des proboscis y compris les crochets $112 \mu$. Longueur des bulbes $633 \mu$; diamètre des bulbes $178 \mu$. Longueur du velum $429 \mu$; largeur du velum antérieurement $594 \mu$, postérieurement $561 \mu$. Longueur du segment antérieur de l'appendix $858 \mu$ (dont 429 dépassent le velum). Longueur du segment postérieur de l'appendix $264 \mu$. Organe sensoriel $70 \times 50 \mu$.

Les crochets ont une longueur d'environ $32 \mu$ (sauf à la base des proboscis où quelques-uns sont plus petits) ; ils sont allongés en forme de serpe et tous de même forme ; leur bord inférieur présente, en son milieu, une légère protubérance dentiforme (d'où le nom spécifique); ils sont disposés en spirale ascendante de gauche à droite tout autour du proboscis, comme chez les Homéacanthes (sous-ordre Atheca).

Il s'agit donc d'une espèce homéacanthe. Les crochets sont pleins, comme chez les homéacanthes à quatre bothridies (alors qu'ils sont creux chez les autres Homéacanthes, les Hétéracanthes et les Pécilacanthes). Aucun proboscis n'est entièrement évaginé ; néanmoins, il semble que les crochets soient insérés suivant quatorze lignes longitudinales. Il est remarquable que l'appendix, ébauche du futur strobile, continue à s'accroître pendant la vie planctonique. 


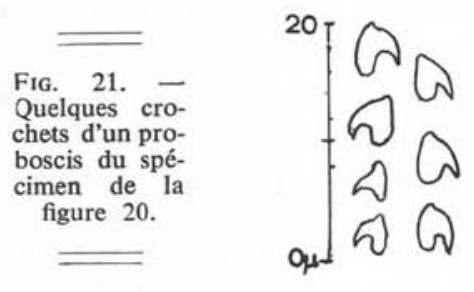

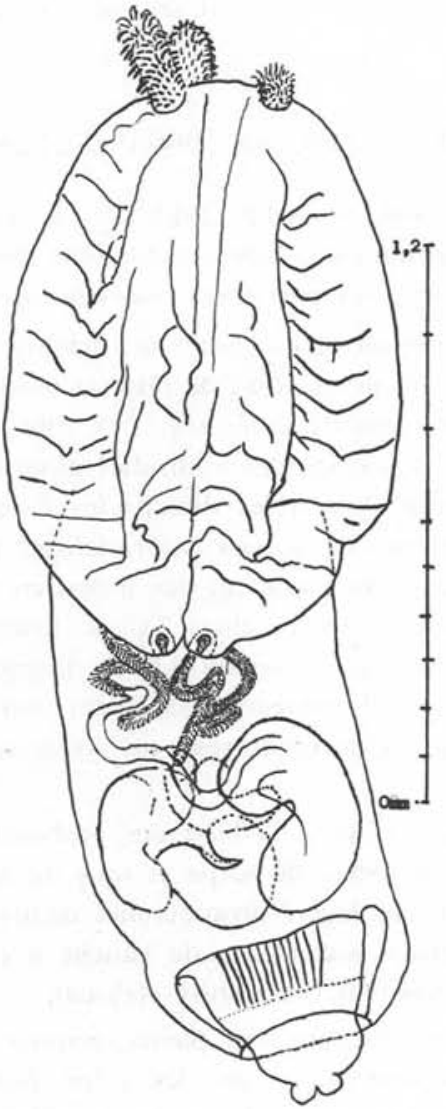

FIG, 23. - Même espèce. Individu vu de face. La pars metabothridïalis est très contractée.

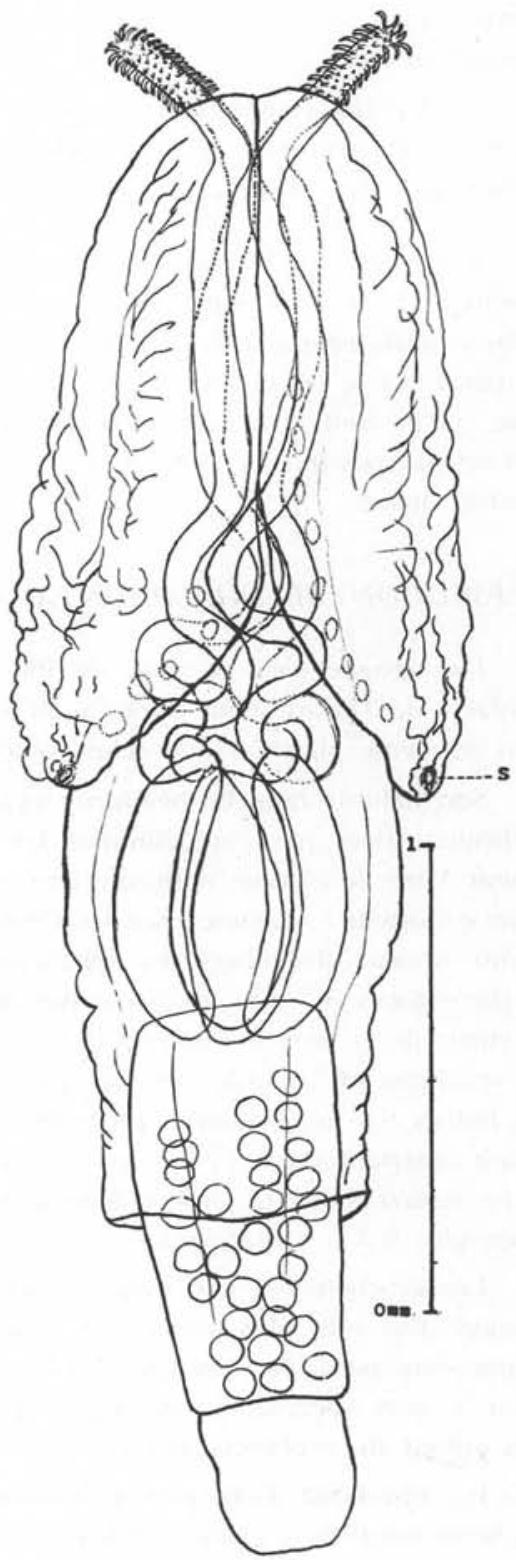

FIG. 22. - Postlarve: Pseudonybelinia odontacantha R.-Ph. Dollfus. Vue latérale. Même provenance. 


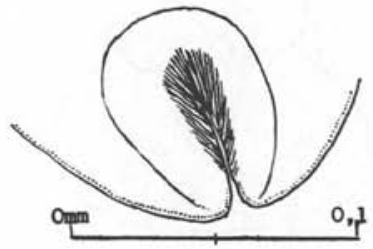

Fig. 24. - Organe sensoriel, postlarve de la figure 23 .

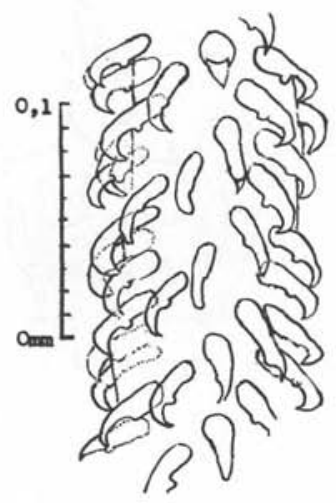

FIG. 25. - Armement d'un proboscis du spécimen de la figure 23 . Vue latérale, partie basale.

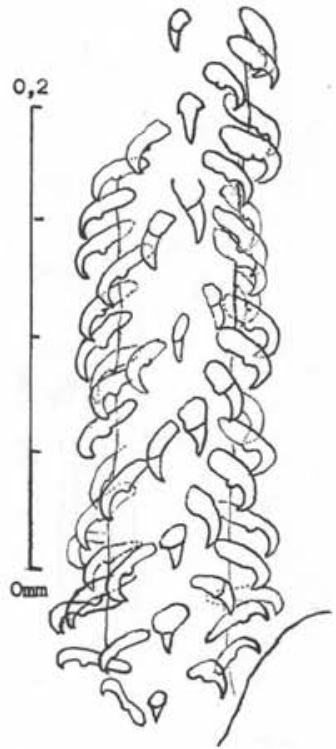

FIG. 26. - Armement d'un proboscis du spécimen de la figure 23 . Vu par la face bothridiale.

D) PARANYBELINIA OTOBOTHRIOIDES R. Ph. Dollfus (fig. 27-31).

J'ai désigné sous ce nom (R.-Ph. Dollfus, 1966, p. 2612, 2615, fig. 2-3) une postlarve de Tétrarhynque dont j'ai eu à ma disposition de nombreux individus prélevés dans le même plancton que les larves et postlarves ci-dessus décrites. Le scolex a un habitus rappelant quelque peu celui des Otobothridiens (d'où le nom spécifique). Comme chez ceux-ci, il y a deux bothridies. Chaque bothridie présente, sur son bord postérieur libre, une fossette sensorielle, comme chez l'espèce précédente. Le spécimen choisi pour type a une longueur de $1,2 \mathrm{~mm}$, y compris le prolongement caudal, une plus grande largeur de $0,31 \mathrm{~mm}$ au niveau du bord postérieur des bulbes. Pars bothridialis $0,19 \mathrm{~mm}$, pars vaginalis $0,18 \mathrm{~mm}$, pars bulbosa $0,86 \mathrm{~mm}$, pars metabothridialis (partie ne dépassant pas le velum) $0,733 \mathrm{~mm}$; velum $0,13 \mathrm{~mm}$. Diamètre des proboscis, sans les crochets $16 \mu$, avec les crochets $29,3 \mu$. Prolongement caudal (compté à partir du bord postérieur du velum) $0,7 \mathrm{~mm}$. Les dimensions des différentes parties du scolex sont les mêmes, jusqu'au bord postérieur du velum, chez tous les individus; sauf une petite variation en ce qui concerne les bulbes contractiles; ils sont brièvement ovales $(80 \times 53 \mu)$ chez l'individu de la figure 27 , moins brièvement ovales $(86,6 \times 60 \mu)$ chez celui de la figure 28 , et ovales allongés $(103 \times 36,6 \mu)$ chez celui de la figure 29 . La longueur du prolongement postérieur, prolifération de l'appendix, varie beaucoup selon les individus. 

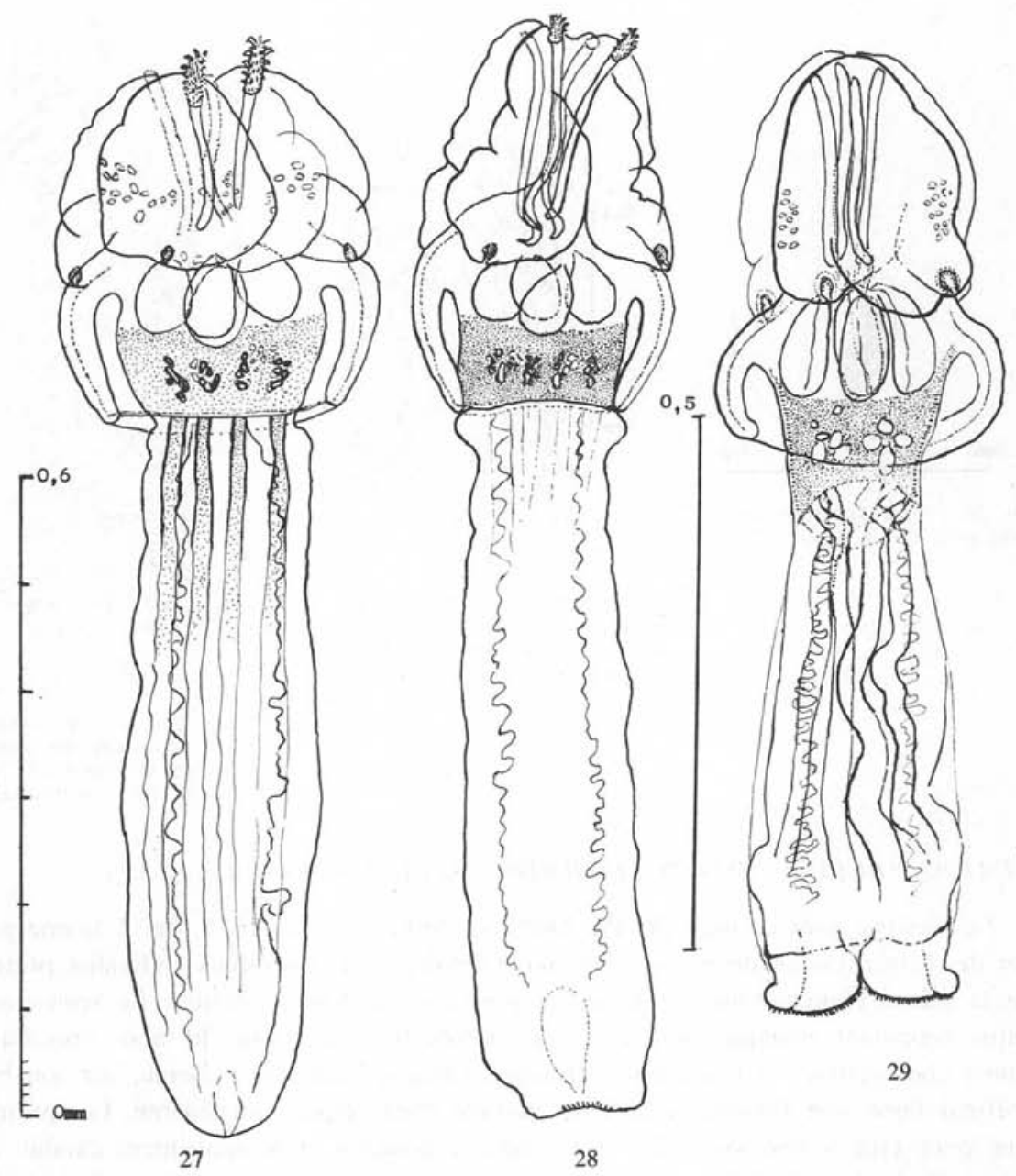

FIG. 27-29. - Postlarve: Paranybelinia otobothrioides. R.-Ph. Dollfus. Habitus de trois individus. Même provenance.

Les crochets des proboscis sont en forme d'arceau et pleins, tous de la même forme, insérés en spirale ascendante de gauche à droite, sur environ douze lignes longitudinales; ils sont longs d'environ $12 \mu$ sur les côtés du proboscis, vus de profil ; peut-être ceux des faces bothridiale et antibothridiale sont-ils un peu moins longs (environ $9 \mu$ ); ceux de la base sont nettement plus petits (fig. 31 ).

Cette postlarve, comme la précédente, malgré ses deux bothridies et ses fossettes sensorielles, appartient aux Homéacanthes (sous-ordre Atheca). Je rappelle que les crochets ne sont jamais en spirale complète chez les Hétéracanthes et Pécilacanthes. 
E) LaRve de position systématique incertaine (fig. 32-33).

De cette larve, j'ai examiné cinq individus colorés au carmin, mesurant, après léger aplatissement: $1,09 \times 0,64 ; 0,85 \times 0,60 ; 0,84 \times 0,69 ; 0,82 \times 0,71 ; 0,79 \times$ $0,56 \mathrm{~mm}$. Le corps est ovale, peu épais. Antérieurement se trouve un organe adhésif à faible musculature, mesurant transversalement 0,25 à $0,35 \mathrm{~mm}$. Immédiatement en

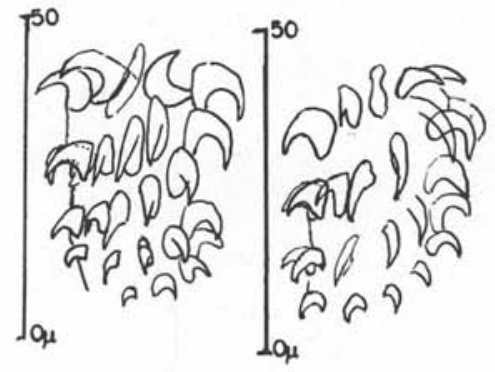

Fig. 30-31. - Armement de la partie basale de deux proboscis du spécimen de la figure 28 .

30

31

FIG. 32-33. - Larve de position systématique indéterminée. Même provenance. Deux individus, Même échelle.

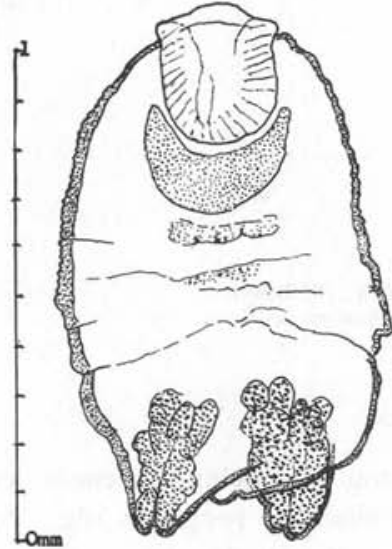

32

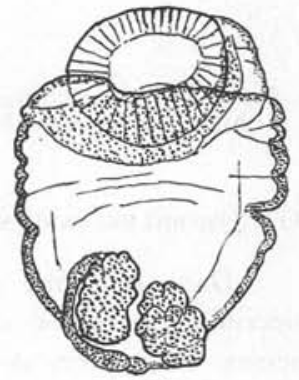

33

arrière, il y a une formation en croissant, plus colorée que le parenchyme environnant. A l'extrémité postérieure sont deux volumineux organes glandulaires, généralement bien séparés l'un de l'autre, dans des plans différents. Chez un des cinq individus, l'extrémité postérieure du corps présente deux protubérances, occupées chacune par la partie postérieure d'un des organes glandulaires.

Cette larve est énigmatique; elle ne peut pas être rapportée avec certitude à un groupe de Cestodes de la faune marine. 
F) Postlarve de Phyllobothrien (fig 34-35).

Cette postlarve, représentée par plusieurs spécimens, comprend un scolex et un prolongement postérieur de longueur variable, mais court. Un individu mesure $0,5 \times$ $0,23 \mathrm{~mm}$, il présente une ventouse apicale de $54 \mu$ de diamètre. Un autre individu mesure $0,369 \times 0,759 \mathrm{~mm}$, sa ventouse apicale a un diamètre de $30 \mu$ (fig. 34-35).

Il s'agit d'une forme jeune, qui a dépassé le stade plérocercoïde, n'ayant conservé aucun attribut larvaire. Le prolongement postérieur est évidemment la première ébauche du futur strobile.
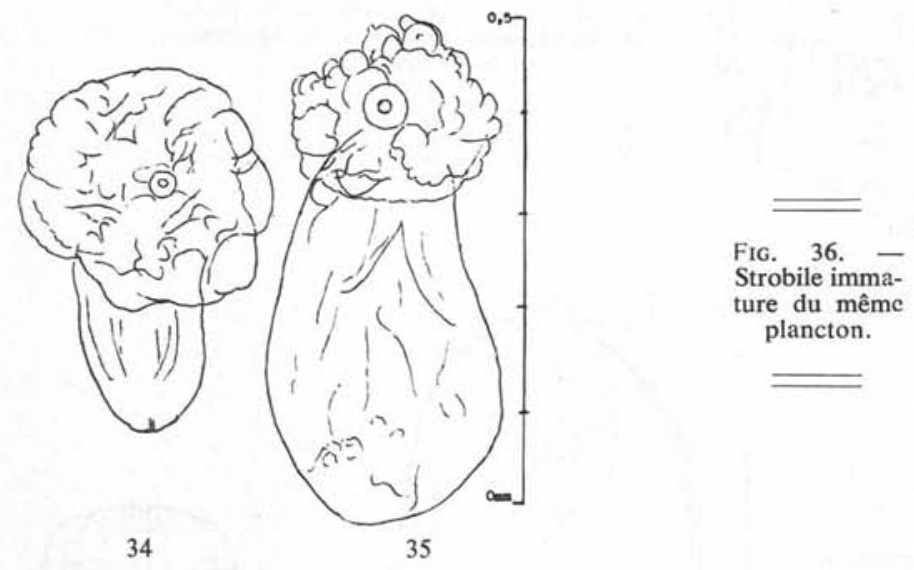

FIG. 34-35. - Postlarve d'un Phyllobothrien indéterminé. Même provenance.

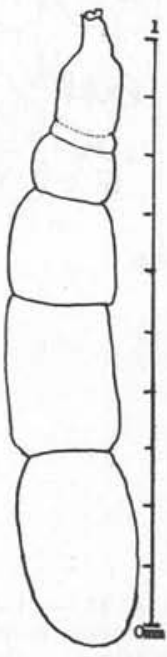

G) Strobiles Sans SCOLEX.

Dans le même plancton que celui contenant les larves et postlarves ci-dessus décrites, j'ai trouvé des chaînes de proglottis (fig. 36); aucune n'est attenante à un scolex. Le nombre des proglottis est de cinq à sept; ils sont tous immatures et aucun organe n'est visible.

H) Larve de Tétraphylle fixée sur un Distome parasite interne de ChaetoGNATHE.

R.-Ph. Dollfus, M. Anantaraman et R.-V. Nair (1955, p. 525, fig. 4, « scolex» fixé sur une métacercaire; fig. 5, même larve «scolex» plus fortement grossie) ont signalé la présence de larves du type «scolex», d'un Tétraphylle indéterminé, fixées sur une métacercaire d'Accacoeliidae, parasite interne de Sagitta inflata Grassi 1881, du plancton de Madras. La fixation de la cercaire avait lieu par le myzorhynchus. 
sphérique avec un diamètre de $20 \mu$. Longueur de la larve $62 \mu$, largeur maximum $40 \mu$, diamètre des ventouses $19 \mu$. Les quatre ventouses latérales sont uniloculaires, à contour circulaire, sessiles.

I) Larve de Tétraphylle chez un Copépode libre.

S. Krishnaswamy (1950, p. 76, fig. 3), chez une ㅇ d'Undinula vulgaris (Dana 1852) du plancton de Madras, a observé la larve du type «scolex », d'un Tétraphyllide indéterminé. Plus de 500 de ces larves se trouvaient dans le Copépode; elles étaient longues de $40 \mu$, pourvues de ventouses latérales sessilles et d'un grand myzorhynchus.

J) LaRves de Tétraphylle et de Tétrarhynque obTenues expérimentalement CHEZ UN COPÉPODE LIBRE.

Dans la nature, aucun Cestode n'est connu chez le Copépode harpacticide Tigriopus fulvus (S. Fischer, 1860), qui habite les flaques d'eau de mer des rochers de la zone supérieure des marées, mais N.W. Riser en a obtenu l'infestation expérimentale par des coracidies d'une espèce de Tétraphylle et d'une espèce de Tétrarhynque. Des œufs d'Acanthobothrium hispidum N.W. Riser 1955 furent mis en présence de Tigriopus; les coracidies ingérées donnèrent, dans l'hémocèle, des procercoïdes avec cercomère portant deux paires de crochets (Riser, 1956, p. 121, 123, fig. 4, procercoïde quinze jours après l'infestation).

Des œufs de Lacistorhynchus tenuis (P. J. Van Beneden 1958) (pondus segmentés) furent placés en eau de mer et donnèrent des coracidies contenant une oncosphère à trois paires de crochets; ces coracidies furent ingérées par des Tigriopus! quelques heures après, les oncosphères furent observées dans les Copépodes; dans un seul de ceux-ci, Riser compta 19 larves ; ces larves s'allongèrent et devinrent des procercoïdes, mais ceux-ci ne présentaient pas de cercomère ; une de ces larves, le $41^{\circ}$ jour, mesurait $0,3 \times 0,09 \mathrm{~mm}$. Deux autres expériences, avec des œufs du même Tétrarhynque et des Tigriopus, donnèrent les mêmes résultats. (Riser, 1951, p. 26 ; 1956, p. 121-123, fig. 1: oncosphère, fig. 2 : coracidium contenant une oncosphère, fig. $3:$ procercoïde dans l'hémocèle de Tigriopus 21 jours après l'infestation, fig. 5: idem 26 jours après l'infestation).

Il est évident que les Tigriopus, dans la nature, ne sont pas hôte intermédiaire de Tétraphylles ou de Tétrarhynques, mais j'ai estimé que les expériences de Riser devaient être mentionnées à propos des Cestodes d'invertébrés marins.

K) LARVE PRÉSumée de Cestode dans un CRUStacé indéterminé.

W. Gordon Fields (1965, p. 31) relate qu'il a observé un organisme, apparemment une larve de Cestode, dans un appendice de crustacé, de l'estomac d'un Loligo opalescens Berry, de la côte ouest des Etats-Unis. 


\section{L) Larves de Tétraphylles chez des CÉphalopodes.}

J. A. Stevenson (1933, p. 38) a trouvé en abondance dans le tube digestif des Loligo peali Le Sueur, $\hat{o}$ et $q$, à St Andrews (Nouveau Brunswick), une larve de Tétraphylle qui a été identifiée à Phyllobothrium loliginis (J. Leidy, 1887) par H. A. Baylis. D'après Edwin Linton (1922, p. 15), cette larve est celle de Phyllobothrium tumidum Edwin Linton 1922.

F. A. Aldrich (1964, p. 16-17), étudiant la biologie des Illex illecebrosus Le Sueur 1821, de Terre-Neuve, a remarqué que, dans le tube digestif, chez les individus de Placentia Bay, les plérocercoïdes de Phyllobothrium étaient beaucoup moins nombreux que chez ceux de Trinity Bay et Conception Bay; cela montre qu'il s'agit de populations différentes et semble bien contredire l'opinion de H. J. Squires (1957), pour qui tous les Illex illecebrosus Le Sueur de l'ensemble de Terre-Neuve appartiennent à une seule population.

Les observations de H. J. Squires (1957) sur les Phyllobothrium et Dinobothrium parasites de cet Illex de Terre-Neuve, ont été rappelées par F. A. Aldrich (1964, p. 12). Comme l'on suggère que les Euphausiacés sont premier hôte pour Dinobothrium et comme les Illex ne se nourrissent d'invertébrés qu'au début de leur vie, il faut admettre, remarque Aldrich, que l'infestation persiste pendant une longue durée de la vie des Illex.

W. Gordon Fields (1965, p. 30) relate que l'on trouve occasionnellement des plérocercoïdes de Tétraphylles dans le caecum ou ailleurs, chez Loligo opalescens S. S. Berry 1911, espèce vivant sur la côte ouest des Etats-Unis (principalement Californie et Washington) et atteignant au nord la Colombie britannique. Ces plérocercoïdes, dit Fields, remuent très activement s'ils sont dérangés ou transportés en eau de mer.

Quelques informations sur les recherches de N.W. Riser (1949, Unpublished $\mathrm{Ph}$. D. Thesis) sont données par Fields (1965, p. 30-31). D'après ce dernier, Riser mentionne deux types de plérocercoïdes de Tétraphylles chez les Loligo opalescens Berry, à Monterey (Californie) et chez les Dosidicus gigas (Orbigny) à Corona del Mar (Californie). Ces deux types de plérocercoïdes ont des phyllidies en forme de feuilles portant antérieurement un acétabulum et sont pourvus d'une ventouse apicale. Ces larves, en arrière de la zone de prolifération, portaient un appendice parenchymateux. Quelques-unes, de Loligo opalescens Berry, étaient petites et grêles, mais Riser a aussi trouvé, d'après Fields, un grand et robuste plérocercoïde, correspondant à la description de Pelichnibothrium speciosum Monticelli 1889, dans Dosidicus, Loligo et le poisson Alepisaurus ferox Lowe 1833, Téléostéen de l'ordre des Iniomi, bathypélagique et occasionnellement côtier.

Les renseignements publiés par N.W. Riser (1956, p. 31) sont un peu différents : Riser mentionne, chez Alepisaurus ferox Lowe, de Carmel (Californie), d'une part de grands plérocercoïdes qu'il rapporte à Pelichnibothrium speciosum Monticelli 1889 , d'autre part de petits plérocercoïdes qu'il rapporte à Pelichnibothrium caudatum Zschokke et Heitz 1914, et il ajoute: de semblables larves étaient présentes dans le caecum de Dosidicus gigas (Orbigny) de Corona del Mar (Californie) et de Loligo opa- 
lescens Berry de Monterey Bay (Californie). Dans cette publication de février 1956, Riser ne dit rien d'autre concernant ces parasites de Céphalopodes ; il y décrit \& Pelichnibothrium speciosum, plerocercoid larva », mais d'après des spécimens de l'intestin et du caecum d'Alepisaurus ferox Lowe, de Carmel (Californie). D'après la description, ces spécimens ne devraient pas être considérés comme larvaires : ils ont tous les caractères d'adultes, ils sont pourvus d'un strobile dont les proglottis contiennent un appareil génital complet (testicules, ovaire, atrium génital, vitellogènes, poche du cirre, cirre, etc...), mais ils ne produisent pas d'œufs. Placés dans une solution de peptone, à la température du laboratoire, les proglottis se sont séparés, les testicules ont atteint la maturité, l'utérus s'est rempli de cellules vitellines émises par déhiscence, mais des œufs n'ont pas apparu. A mon avis, si les spécimens étudiés par Riser ne sont pas des adultes incomplètement mûrs, ce sont des postlarves à progénèse incomplète.

D'autres plérocercoïdes se déplaçant activement ont aussi été trouvés, dit W. Gordon Fields (1965, p. 31) chez Loligo opalescens Berry ; ils ont la forme du corps et l'appendice caudal semblables à ceux décrits plus haut, mais les bothridies, observées sur le vivant, sont dépourvues d'acétabulum antérieur, et biloculaires; leur bord libre et leur septum ne sont pas ondulés, n'ont pas de plis et leur largeur est uniforme. Ces bothridies, remarque Fields, ressemblent à celles du plérocercoïde figuré par $\mathrm{Ch}$. Joyeux et J. G. Baer (1936, p. 539, fig. 569 B), sauf que les loculi antérieurs ont un diamètre quelque peu plus petit que celui des loculi postérieurs.

W. Gordon Fields $(1965$, p. 31) relate que l'on trouve occasionnellement des plérocercoïdes encapsulés dans la paroi musculaire de l'estomac ou de l'œsophage des Loligo opalescens Berry. Ces plérocercoïdes, dit Fields, ressemblent beaucoup à ceux figurés par Ch. Joyeux et J. G. Baer (1936, fig. 559, Plerocercoides aequoreus Wundsch) et par Wardle et McLeod (1952, fig. 105, «Scolex pleuronectis»). Ils sont attribués à Phyllobothrium sp. par N. W. Riser (communication personnelle à W. G. Fields).

M) Larves de divers Cestodes chez des Lamellibranches.

Aux Hawaii, chez Crassostrea virginica (Gmelin), espèce d'huître américaine introduite aux Hawaii, Albert K. Sparks (1963, p. 284-288, fig. 1-3) a trouvé la larve d'un Tylocephalum (Lecanocephaloidea) dans des kystes fibreux mesurant environ $100 \times 120 \mu$, de la région des diverticules digestifs, quelquefois dans la paroi de l'intestin ou des tubules digestifs, mais principalement dans le tissu conjonctif entre les diverticules digestifs et sous l'intestin.

Examinant histologiquement des Crassostrea virginica (Gmelin) transplantés de l'Apalachicola Bay (Florida) à la Chinesteague Bay (Maryland), Richard W. Burton (1963, p. 61-62) reconnut qu'elles étaient abondamment parasitées par une larve qu'il attribua à Tylocephalum. Ces larves mesuraient de 105 à $231 \mu$, la plus grande $210 \times$ $231 \mu$, et étaient contenues dans des kystes épais, apparemment formés par l'huître. Pour la plupart, elles se trouvaient dans le tissu conjonctif environnant l'épithélium du gros intestin, plusieurs étaient dans les tissus des branchies et une dans un palpe.

Une larve similaire est probablement celle trouvée dans des Penaeus de Floride (voir R. Ph. Dollfus, 1964, p. 371-372) et qui fut soumise à S. Yamaguti par R. F. Hutton pour savoir s'il s'agissait d'un Cestode. Des spécimens vivants de cette larve 
ont été étudiés par F. Sogandarès-Bernal (communication personnelle du 16 mars 1965), qui a définitivement reconnu qu'il s'agissait d'un Cestode et il y a vu des corpuscules calcaires.

Dans « 1965 Research in Fisheries », rapport publié par le « College of Fisheries », Université de Washington, contribution $\mathrm{n}^{\circ} 212$, march 1966, p. 23, on lit qu'une larve d'Echeneibothrium a été trouvée chez Venerupis staminea Conrad, de la baie Humboldt (Californie). Cette larve a été étudiée par A. K. Sparks et K. K. Chew (1966, p. , fig. 1-2) ; elle était répandue dans tous les tissus du corps de l'hôte et contenue dans des kystes réactionnels à paroi épaisse (fig. 37-38) (4). Les quatre bothridies étaient subdivisées en dix séries d'aréoles par des rides transversales, le myzorhynchus « dome like » montrait une « internal spherical muscle mass and a terminal opening in the center».

L'adulte correspondant n'a pas, jusqu'à présent, été spécifiquement identifié. Sparks et Chew ont comparé leur larve à Echeneibothrium minimum P. J. Van Beneden 1850

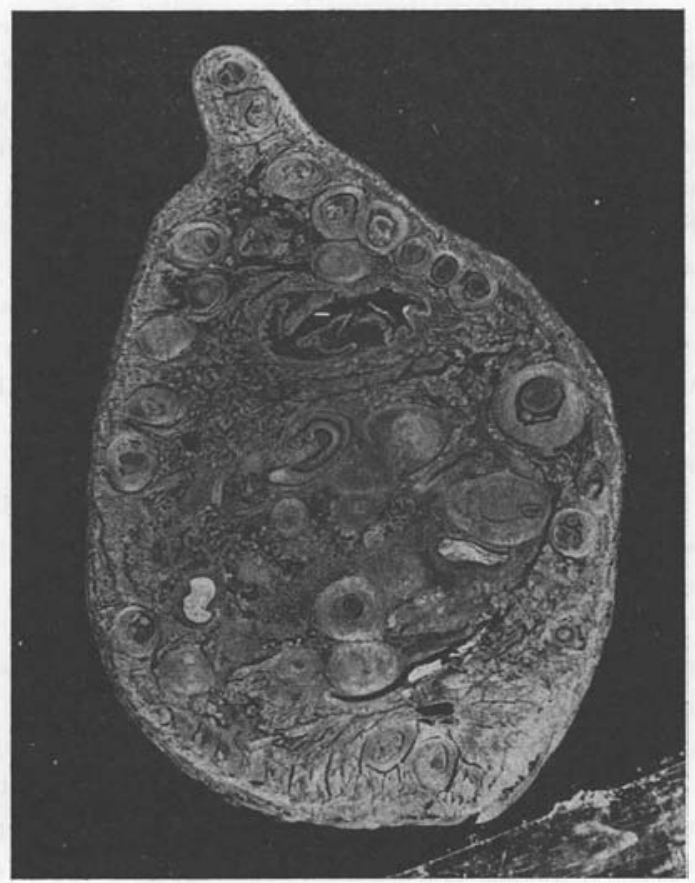

FIG. 37. - Larves d'un Echeneibothrium sp. dans des xénokystes (capsules réactionnelles). Coupe transversale dans Venerupis (Paphia) staminea Conrad. Baie de Humboldt (California). Photographie par A. K. Sparks \& K. K. Chew.

(4) Tous mes remerciements au Professeur Albert K. Sparks pour ces deux photographies. 
et à E. myzorhynchus J. H. Hart 1936 (qui, pour Riser, 1955, est une espèce composite) ; ils ont même tenté une comparaison, d'après le myzorhynchus, avec Discobothrium fallax P. J. Van Beneden 1870 ; mais, après discussion, aucune de ces espèces n'a été prise en considération.

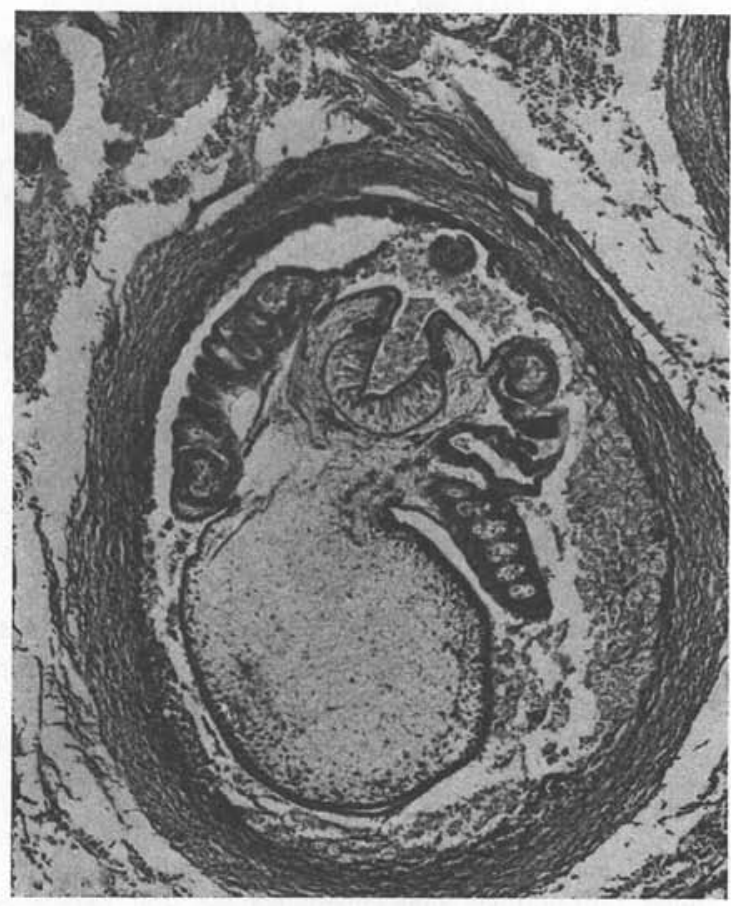

FIG. 38. - Coupe d'un des xénokystes de la coupe de la figure 37 .

\section{Bibliographie}

AldRICH (Frederick A.), 1964. - Observations on the Newfoundland bait squid (Illex illecebrosus. Lesueur 1821) and the netting of squid in Newfoundland bays. Memorial University of Newfoundland. Department of Biology, nov., p. 1-22, fig. 1-6 (tirage ronéotypé).

BURTON (Richard W.). 1963. - A Cestode microparasite of the oyster, Crassostrea virginica from Florida. Proceedings annual Shellfish mortality Conference. Bureau of commercial fisheries biological Laboratory, Oxford (Maryland), Jan. 29-30, p. 61-62.

Dollfus (Robert Ph.), 1966. - Organismes dont la présence dans le plancton marin était, jusqu'à présent, restée ignorée: larves et postlarves de Cestodes Tétrarhynques. Comptes rendus hebdom. Acad. Sciences, Paris, t. CCLXII, $\mathrm{n}^{\circ} 25$, série D, séance du 12 juin, p. 2612-2615, fig. 1-4. 
Dollfus (Robert Ph.), Anantaraman (Mahadena) \& Nair (R. Volappan), 1954. - Métacercaire d'Accacoeliidé chez Sagitta inflata Grassi et larve de Tétraphylle fixée à cette métacercaire. Annales Parasitol. hum. et comp., t. XXIX, p. 521-526, fig. 1-5.

FIELDS (W. Gordon), 1965. - The structure, development, food, relations, reproduction, and life history of the Squid Loligo opalescens Berry. State of California. The Resources Agency, Department of Fish and Game, Fish Bulletin 136, p. 1-108, fig. 1-59.

KRIShNaSwamy (S.), 1950. - A note on the parasites of free living copepods of Madras. Madras University Journal, ser. B., v. XX, p. 75-78, pl. fig. 1-6.

McPHAIL (J. D.), editor, 1966. - Epizooties in experimental marine shellfish population. Research in Fisheries... 1965. College of Fisheries, Fisheries Research Institute. University of Washington, Seattle, Washington 98105 . Contribution $\mathrm{n}^{\circ} 212$, march 1966, p. 22.

Riser (Nathan W.), 1951. - The Procercoid larva of Lacistorhynchus tenuis (van Beneden 1858). Journal of Parasitol., v. XXXVII, $\mathrm{n}^{\circ}$ 5, Section 2, p. 26.

-, 1956. - Observations on the plerocercoid larva of Pelichnobothrium speciosum Monticelli 1889. Journ. of Parasitol., v. XLII, $\mathrm{n}^{\circ}$ 1, p. 31-33, fig. 1.

-, 1956. - Early larval stages of two Cestodes from Elasmobranch Fishes. Proc. helminthol. Soc. Washington, v. XXIII, $\mathrm{n}^{\circ}$ 2, july, p. 120-124, fig. 1-5.

SPARKS (Albert K.) 1963. - Infection of Crassostrea virginica (Gmelin) from Hawaii with a larval tapeworm, Tylocephalum. Journ. Insect Pathology, v. $\mathrm{V}, \mathrm{n}^{\circ} 3$, sept., p. 284-288, fig. 1-3.

SPARKS (Albert K.) \& CHEW (Kenneth K.) 1966. - Gross Infestation of the Littleneck Clam (Venerupis staminea) with a larval Cestode (Echeneibothrium sp.). Journ. Invertebrata Pathology, sept. 1966, p. , photo 1-2.

Stevenson (J. A.) 1933. - Squid (Loligo pealii) at St-Andrews, N.B. - Annual Report biological Board of Canada for the year 1932, Ottawa, p. 37-38.

Note AJOUtée LORS DE LA CORRECTION DES ÉPREuves. - Depuis la rédaction de cette $7^{\circ}$ contribution, quelques informations supplémentaires me sont parvenues.

$1^{\circ}$ Le Dr M.-C. Mercer m'a envoyé des larves de Tentacularia coryphaenae, L. A. G. Bosc 1802 qu'il a trouvées à Terre-Neuve dans un Cucioteuthis unguiculatus (Molina 1782) (fam. Octopodoteuthidae). Il s'agit de véritables larves encore dans leur étui larvaire (blastocyste) ; ce sont des larves âgées, mais pas des post-larves.

$2^{\circ}$ Martin Weinstein, dans le Rapport annuel pour 1965 de la Station de Biologie marine de Grande-Rivière [Canada] relate (1966, p. 56), qu'il a trouvé « a single tapeworm plerocercoid of the Scolex polymorphus type $»$ chez une Sagitta elegans Verrill, du golfe du SaintLaurent.

$3^{\circ}$ Maryvonne Hamon (1956, p. 472, Bull. Mus. Nat. Hist. Nat., Paris, $2^{\circ}$ s., t. XXVIII, $\mathrm{n}^{\circ}$ 5) examinant des Sagitta du plancton de la Baie de Nhatrang (Annam) a relaté qu'elle avait trouvé «des cysticercoïdes (semble-t-il) chez $S$. inflata, bedoti, et neglecta ».

$4^{\circ}$ M. B. B. Grey (1931, p. 65-66, fig. 12) dans le Proceed. Royal. Soc. Queensland (vol. XLII, for 1930, $\mathrm{n}^{\circ} 6,29-1-1931$ ), mentionne un «cysticercus in body coelome» chez Sagitta fridrici des Iles de la Société.

(Muséum National d'Histoire Naturelle, 57, rue Cuvier, Paris- $V^{\circ}$ ). 